\title{
Prevalence and Gender Ratio of Dyslexia in Greek Adolescents and Its Association with Parental History and Brain Injury
}

\author{
F. Vlachos ${ }^{1, *}$, E. Avramidis ${ }^{1}$, G. Dedousis ${ }^{2}$, M. Chalmpe ${ }^{1}$, I. Ntalla ${ }^{2}$, M. Giannakopoulou ${ }^{3}$ \\ ${ }^{1}$ Department of Special Education, University of Thessaly, Volos, Greece \\ ${ }^{2}$ Department of Nutrition and Dietetics, Harokopio University, Athens, Greece \\ ${ }^{3}$ Department of Nursing, National and Kapodistrian University of Athens, Athens, Greece \\ *Corresponding author: fvlachos@uth.gr
}

Received December 21, 2013; Revised January 18, 2013; Accepted January 28, 2013

\begin{abstract}
Dyslexia is the most common and carefully studied of the learning disabilities in school-age children. It is characterized by a marked impairment in the development of reading skills, and affects a large number of people. The prevalence of dyslexia shows considerable cross-national variation. Additionally, a plethora of research studies have indicated that there are more boys than girls with reading difficulties. The aim of this study was to identify the frequency and gender ratio of dyslexia in a sample of Greek adolescents and their siblings. 598 secondary school students (Mean age 13.33, SD = 1.49) who attended mainstream public schools participated in this study. The prevalence of dyslexia in this study was estimated at $5.52 \%$, a finding consistent with the data from other countries with "pure" orthographies. Statistical analysis revealed significant differences in the prevalence of dyslexia between gender (7.6\% male, 3.8\% female), which means that boys were twice as likely to be identified as dyslexic than their female peers. Additionally, statistically significant differences were observed between dyslexics who had a parent suffering from dyslexia (15.1\%), compared to normal readers (1.8\%), but no differences were observed between the two groups as for the frequency of brain injuries. Overall, our findings are in accordance with the results of previous national studies indicating the universal existence and the biological basis of this developmental disability.
\end{abstract}

Keywords: Dyslexia, Prevalence, Gender

\section{Introduction}

Developmental dyslexia is the most common of the learning disabilities in school-age children. It has a biological and genetic basis characterized by a marked impairment in the development of reading skills and affects a large number of people [1,2]. Reading disabilities are common, with prevalence estimates ranging from 5 to $15 \%$ of the school-aged population, depending on how the disability is defined [3]. For example, in Australia, it is believed that up to $20 \%$ of the student population struggle to learn to read, while approximately $10 \%$ of those experience substantial reading difficulties [4]. Other studies have reported an incidence of reading disability of up to $11 \%$ [5]. Some authors [6] have concluded that this reading disability varies from 5 to $15 \%$ of the school population depending on the study, whereas others claim that only a small subset of the population (4-6\%) is reading disabled [7]. Another reason accounting for the considerable cross-national variation of the prevalence of dyslexia may be related to differences among languages in the regularity of grapheme-phoneme correspondence. For example, reading disabilities have been noted to be rare in
Japan and to be considerably less frequent in southern Europe than in northern Europe or North America.

Additionally, a significant body of research claims that more boys than girls experience reading problems [8]. This is also supported by a recent review [9] of empirical studies which concluded, that on the whole, more boys than girls are diagnosed with reading disability. Although the degree varies considerably, most studies have reported gender ratios of 3:1 or higher [10]. More recently, it has been suggested [3] that there is male preponderance in reading disabilities, with a ratio of about 1.5:1 which is lower than the historical estimates of about 3-4:1. However, despite the enormous body of evidence, some researchers have reported little or no significant gender differences. For example, a study [11] found no significant gender differences when samples were research-identified as opposed to school-identified, indicating that the greater prevalence of boys with reading disabilities was due to referral bias. Similarly, a longitudinal study [12] reported no significant gender differences in reading disabilities. Finally, a recent study in Greece [13] revealed that dyslexia was more common in boys than girls $(59,7 \%$ vs. $35,1 \%$ ).

The origins of dyslexia are neurobiological with strong evidence for heritability. According to recent reviews of 
the literature $[14,15]$, many studies of the heritability of dyslexia show converging evidence that reading skills have a strong genetic influence. In family segregation studies, the risk of dyslexia is 8 times higher in children where there is a parental history of reading difficulties. Other studies report that $25 \%-60 \%$ of the parents of dyslexic children also display reading difficulties [3].

In another study [16], it has been hypothesized that gender ratios of children with developmental language/learning disorders and their siblings vary as a function of parental impairment. In a family study of 62 children with developmental language/learning disorders, a significant excess of males (44 males and 18 females, a ratio of $2.4: 1$ ) was observed. Additionally, they found that the gender ratio in this referred sample varied as a function of parental impairment: 1.9 : 1, when neither parent was affected; $1.8: 1$, when only the father was affected; $4.0: 1$, when only the mother was affected; and $5.0: 1$, when both parents were affected. Aiming to test the hypothesis, a recent study [17] reported gender ratios for 374 reading-disabled and their 530 siblings included in five independent studies of reading disability. The ratios were tabulated for each study as a function of parental impairment (neither parent affected, mother only affected, father only affected, and both parents affected). Their results revealed a small excess of male probands in referred and clinic samples of reading-disabled children, but not in research identified samples. Interestingly, a Greek study [13] found a significantly higher prevalence of dyslexia in children with a family risk (80\%) contrary to $46.7 \%$ of the contol group.

Although developmental dyslexia is not the result of brain damage [1], the term dyslexia was used in early case studies of individuals with brain injury. More recent studies [13] have also found a significantly higher prevalence of dyslexia in children who had suffered an early brain injury and had received hospital treatment.

This study aimed: (a) to identify the prevalence of dyslexia in a sample of Greek adolescents; (b) to evaluate the gender ratio of dyslexia among these adolescents; (c) to assess the heritability of dyslexia, examining the risk of dyslexia in children with a parental history of reading difficulties as well as testing further Tallal's [16] hypothesis and (d) to explore the possible association of early brain injury with dyslexia.

\section{Method}

\subsection{Survey Instrument}

For the purpose of this study, a questionnaire was designed by the research team. The questionnaire consisted of a demographics section eliciting information about the students' background characteristics and additional sections relating to their academic performance in their school. Moreover, students had to indicate if they had received a statement of dyslexia following assessment at a public or private Centre of Diagnosis, among those included in the lists of the Ministry of Education for the assessment of specific learning difficulties. Additionally, the instrument included two questions requesting students to indicate if they had a history of brain injury and their parents' history of dyslexia respectively. The aims of all sections were clearly stated thus ensuring that the instrument had high 'face' validity. Finally, the appropriateness of all items and the instrument's 'content' validity was confirmed by two expert academics independent to the study.

\subsection{Sampling Procedures and Participants}

Multistage cluster sampling was employed with a view to drawing a nationally representative sample. The process entailed three stages: the sampling of two geographical regions, the sampling of two counties within the selected regions, and the sampling of twelve secondary schools within the selected counties. This final stage also entailed stratification in terms of school location; that is, we randomly chose four urban (located in cities with more than 10,000 inhabitants), four semi-urban (places of 2,500 to 10,000 inhabitants), and four rural schools (areas with less than 2,500 inhabitants). Following this clustering strategy, questionnaires were administered to seventy randomly selected students in each of the twelve selected schools (total sample of 840 students). Completed questionnaires were returned by 598 students (age range 13-18 years; Mean age 13.33; S.D. $=1.49$ ) representing a $71 \%$ return rate. All participants spoke Greek as their first language, and did not have a history of major medical illness, psychiatric illness, or significant visual or auditory impairments according to the medical reports of their schools.

The validity of the study was enhanced through requesting supporting evidence from those students who had reported a diagnosis of dyslexia or a history of brain injury. Further, the study's repeatability was assessed through the Test-Retest technique, which involved 120 resubmissions (20\% of the total sample) and subsequent comparison of the results of the administrations. The remarkably high coefficient of stability $(r=0.96)$ confirmed the study's reliability.

\section{Results}

\subsection{Incidence of Dyslexia in a Sample of Greek Adolescents}

First, we calculated the number of dyslexic adolescents in our sample. As it can be seen in Table 1, 5.5\% of the participating adolescents stated that they were dyslexic:

Table 1. Frequency of Dyslexia

\begin{tabular}{|c|c|c|}
\hline Taving Dyslexia & Frequency & Percent \\
\hline No & 565 & 94,5 \\
\hline Yes & 33 & 5,5 \\
\hline Total & 598 & 100,0 \\
\hline
\end{tabular}




\subsection{Gender Differences in Dyslexia among these Adolescents}

Next, the analysis examined the prevalence of dyslexia between genders. The chi-squared analysis performed revealed a statistically significant association $(\chi 2=4.02, p$ $=.45$ ). Specifically, $7.6 \%$ of the participating boys (21 out of 277) stated that they had been identified as dyslexics as opposed to $3.8 \%$ of the participating girls (12 out of 316 ), which means that boys were twice as likely to be identified as dyslexic than their female peers. These percentages are depicted in Figure 1:

\subsection{The Heritability Hypothesis}

Next, the analysis tested the heritability hypothesis whereby dyslexic adolescents are more likely to have a parent suffering from dyslexia. The chi-squared analysis performed revealed a statistically significant association $\left(x^{2}=22.73, p<.001\right)$. Specifically, $15.1 \%$ of the participating dyslexics had a parent suffering from dyslexia (15.1\%), compared to $1.8 \%$ of the participating normal readers. These percentages are depicted in Figure 2:



Figure 1. Percentages of dyslexic boys and girls

\subsection{The Association of an Early Brain Injury with Dyslexia.}

Additionally, the analysis examined the association between the experience of a brain injury and the subsequent identification of dyslexia. The chi-squared analysis did not reveal a statistically significant association $(x 2=.11, p=.75)$. Specifically, the frequency of brain injuries was rather similar for the two groups i.e. $9.6 \%$ and $7.7 \%$ respectively. These percentages are depicted in Figure 3.

\section{Discussion}

The prevalence of developmental dyslexia in our study's sample of Greek adolescents $(5.52 \%)$ was similar to the ones reported in other countries with "pure" orthographies. More specifically, previous research [18] has reported a general population prevalence of approximately 5\% for dyslexics. Additionally, we found that boys were twice as likely to be identified as dyslexic than their female peers. This finding confirms previous research suggesting that reading disability was about twice as common in boys than girls, irrespective of race, severity of disability, or exclusion of children with attentional disturbances or high activity levels [19]. Our results are also in accordance with a comprehensive review [20] on male vulnerability to reading disability, which concluded that an overall gender ratio of between 1.74:1 and $2: 1$ is most likely to exist. Overall, from the aforementioned studies we could conclude that there is a clear gender bias toward males for the incidence of reading disabilities.

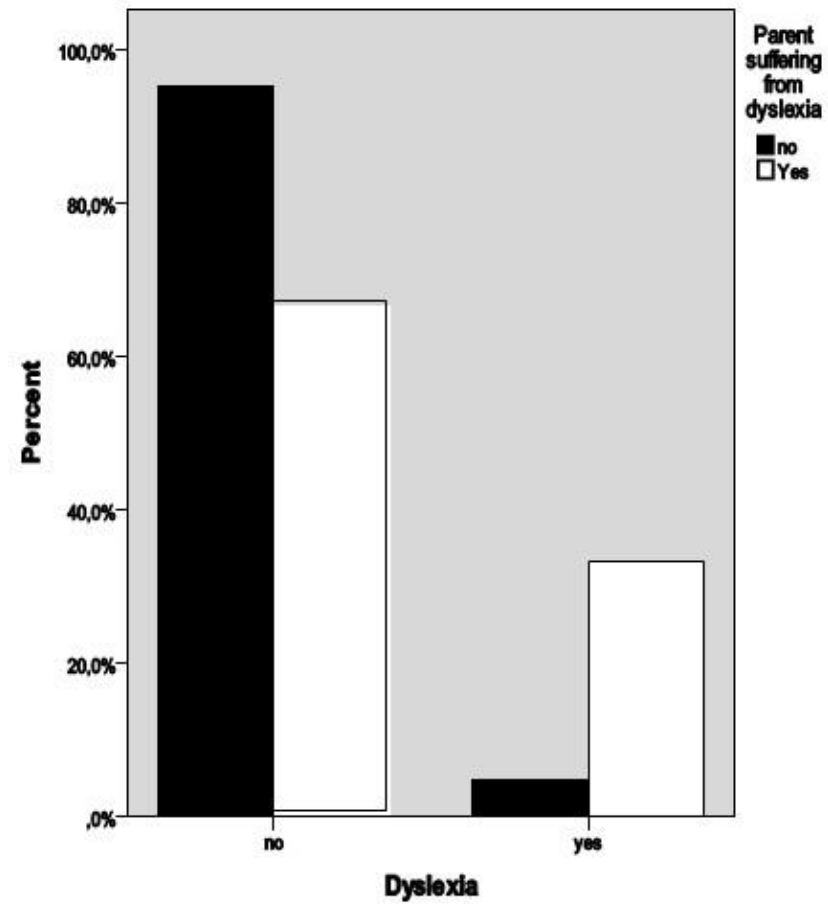

Figure 2. Percentages of parent being dyslexic in the dyslexic and normal reader subgroups

Additionally, our findings showed that children of a dyslexic parent had a significant higher chance of being dyslexics than the children of normal readers, indicating that there is a strong familial effect upon reading abilities. However, as some authors $[2,14]$ suggest, taking together, the findings from the family risk studies indicate that dyslexia is a multifactorial trait in which basic constitutional (genetic) vulnerabilities (notably in phonological skills) interact with other cognitive skills and environmental factors to produce an increased risk of dyslexia in a continuous way.

Overall, our findings are in accordance with the results of previous national studies $[6,7]$, indicating the universal 
existence and the biological basis of this developmental disability.



Figure 3. Percentages of brain injury in the dyslexic and normal reader groups.

\section{References}

[1] Shastry, B., "Developmental dyslexia: An update," Journal of Human Genetics, 52, 104-109, 2007.

[2] Vlachos, F., "Dyslexia: A synthetic approach to causal theories," Hellenic Journal of Psychology, 7, 205-240, 2010.

[3] Pennington, B., Diagnosing learning disorders: A neuropsychological framework ( 2 nd ed.)., Guilford Press, New York, 2009.

[4] Coltheart, M. \& Prior, M., Learning to read in Australia. Canberra: Academy of the Social Sciences in Australia., 2007.

[5] Katusic, S., Colligan, R., Barbaresi, W., Schaid, D. \& Jacobsen, S., "Incidence of reading disability in a population-based birth cohort 1976-1982," Mayo Clinic Proceedings, 76, 1081-1092, 2001.
[6] Prior, M., Samson, A., Smart, D. \& Oberklaid, F., "Reading disability in an Australian community sample," Australian Journal of Psychology, 47, 32-37, 1995.

[7] Sofie, C. \& Riccio, C., "A comparison of multiple methods for the identification of children with reading disabilities," Journal of Learning Disabilities, 35, 234-244, 2002.

[8] Wheldall, K., \& Limbrick, L., "Do more boys than girls have reading problems?," Journal of Learning Disabilities, 43, 418-429, 2010.

[9] Limbrick, L., Wheldall, K., \& Madelaine, A., "Gender ratios for reading disability: Are there really more boys than girls who are low-progress readers?," Australian Journal of Learning Difficulties, 13, 161 - 179, 2008.

[10] Miles, T., Haslum, M., \& Wheeler, T., "Gender ratio in dyslexia," Annals of Dyslexia, 48, 27-55, 1998.

[11] Shaywitz, S., Shaywitz, B., Fletcher, J., Escobar, M., "Prevalence of reading disability in boys and girls: Results of the Connecticut longitudinal study," Journal of the American Medical Association, 264, 998-1002, 1990.

[12] Siegel, L., Smythe, I., "Reflections on research on reading disability with special attention to gender issues," Journal of Learning Disabilities, 38, 473-477, 2005.

[13] Apostolara, P., Tsoumakas, K., Diomidous, M., \& Kalokerinou, A., "The Correlation between Dyslexia and Social and Demographic Factors in Children of School Age," Nosileftiki, 49, 164-173, 2010.

[14] Vellutino, F., Fletcher, J., Snowling, M., \& Scanlon, D., "Specific reading disability (dyslexia): What have we learned in the past four decades?," Journal of Child Psychology and Psychiatry, 45, 2-40, 2004.



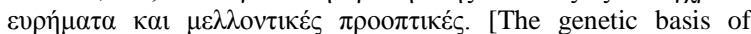
dyslexia: Current findings and future perspectives (in Greek)]". In P. Orfanos (Ed.) The special education in the society of knowledge, Grigoris Publications, Athens, Greece, 2007, 132-141.

[16] Tallal, P., Ross, R. \& Gurtiss, S., "Unexpected sex-ratios in families of language/learning impaired children," Neuropsychologia, 27, 987-998, 1989.

[17] Wadsworth, S., DeFries, J., Stevenson, J., Gilger, J., \& Pennington, B., "Gender ratios among reading disabled children and their siblings as a function of parental impairment," Journal of Child Psychology and Psychiatry, 33, 1229-1239,1992.

[18] Snowling MJ, Muter V, Carroll J, "Children at family risk of dyslexia: a follow-up in early adolescence," J Child Psychol Psychiatr 48, 609-618, 2007.

[19] Flannery, K., Liederman, J., Daly, L. \& Schultz, J., "Male prevalence for reading disability is found in a large sample of black and white children free from ascertainment bias," Journal of the International Neuropsychological Society, 6, 433-442, 2000.

[20] Liederman, J., Kantrowitz, L., \& Flannery, K., "Male vulnerability to reading disability is not likely to be a myth: A call for new data," Journal of Learning Disabilities, 38, 109-129, 2005. 